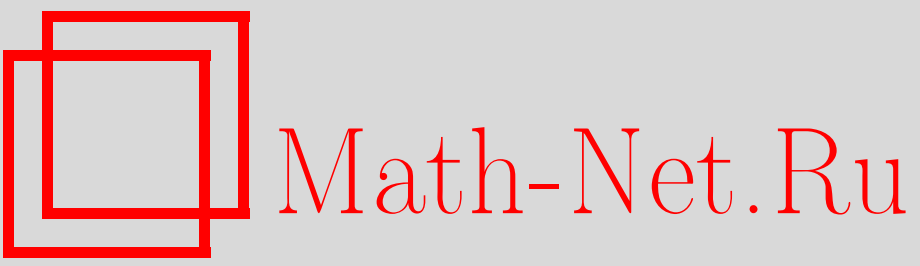

М. Ю. Ковалевский, А. А. Рожков, К теории В-фазы сверхтекучей фермижидкости с триплетным спариванием, ТМФ, 1997, том 113, номер 2, 313-330

DOI: https://doi.org/10.4213/tmf1081

Использование Общероссийского математического портала Math-Net.Ru подразумевает, что вы прочитали и согласны с пользовательским соглашением

http://www.mathnet.ru/rus/agreement

Параметры загрузки:

IP : 54.210 .77 .194

26 апреля 2023 г., 15:22:49 
ТЕОРЕТИЧЕСКАЯ

И МАТЕМАТИЧЕСКАЯ

ФИЗИКА

Том 113, № 2

ноябрь, 1997

М.Ю. Ковалевский* ${ }^{*}$ А.А. Рожков ${ }^{\dagger}$

\title{
К ТЕОРИИ В-ФАЗЫ СВЕРХТЕКУЧЕЙ ФЕРМИ-ЖИДКОСТИ С ТРИПЛЕТНЫМ СПАРИВАНИЕМ
}

\begin{abstract}
Построена термодинамика и дан вывод уравнений гидродинамики сверхтекучей ферми-жидкости с триплетным спариванием. Параметры сокращенного описания сверхтекучая фаза и ортогональная матрица поворота - представлены в терминах оператора параметра порядка. Дано обобщение теоремы о связи плотностей потоков аддитивных интегралов движения на случай локально-равновесных состояний. Детально изучены галилеево- и лоренц-инвариантные физические системы. Получены спектры коллективных возбуждений рассматриваемой ферми-жидкости.
\end{abstract}

\section{1. ВВЕДЕНИЕ}

Открытие явления сверхтекучести Не-ІІ стимулировало теоретические исследования квантовых бозе-жидкостей [1-4]. В последние два десятилетия в связи с открытием сверхтекучести Не-3 интенсивно исследуются квантовые ферми-жидкости [5-10]. Квантовая бозе-жидкость или ферми-жидкость с синглетным спариванием характеризуется скалярным параметром порядка (см., например, $[3-5,9,11])$. В состоянии равновесия в этом случае имеет место спонтанное нарушение симметрии относительно фазовых преобразований. Сверхтекучая ферми-жидкость с триплетным спариванием характеризуется тензорным параметром порядка $[5,10,11]$. Состояние равновесия для нее спонтанно нарушено как относительно фазовых преобразований, так и относительно групп вращения в конфигурационном и спиновом пространствах.

Достаточно сложный параметр порядка в сверхтекучей ферми-жидкости с триплетным спариванием приводит к принципиальной возможности различных наборов равновесных состояний $[9,10,12-14]$, из которых реально обнаружены А-, А - и В-фазы.

При построении гидродинамики сверхтекучих фаз Не-3 обычно исходили из модельных гамильтонианов, представляющих собой квадратичную форму от гидродинамических параметров [15-17]. Дальнейшие исследования $[6,18,19]$ показали, что учет только этих слагаемых недостаточен. Поэтому в этих работах было предложено устранить возникшие расхождения путем учета в исходном гамильтониане слагаемых высших порядков (третьего и вьше), связанных с существованием взаимодействия между спиновыми и орбитальными степенями свободы. Как будет показано в настоящей работе, при

* Харьковский физико-технический институт, Харьков, Украина

${ }^{\dagger}$ Научный физико-технологический центр НАН и МО Украины, Харьков, Украина 
последовательном полуфеноменологическом выводе уравнений гидродинамики нет необходимости использовать конкретный модельный вид функционала энергии.

Вопросам описания состояния равновесия и получения уравнений гидродинамики нормальных и квантовых жидкостей на основе принципов статистической механики посвящены работы [20-25], основу рассмотрения которых составляют гипотеза сокращенного описания и концепция квазисредних [26].

В качестве метода исследования в данной работе выбран ферми-жидкостный подход $[1,5,27]$. Этот метод ранее применялся для описания сверхтекучей ферми-жидкости с синглетным спариванием [27-29]. При таком же подходе в работах [30, 31] рассматривались магнитные системы со спонтанным нарушением симметрии относительно спиновых вращений. Изучая сверхтекучую ферми-жидкость с триплетным спариванием, мы не требуем обрашения в нуль равновесных значений вектора спирали и спина, что обычно имеет место в В-фазе Не-3, а рассматриваем более широкий класс возможных квантовых жидкостей.

Впервые предположение о существовании релятивистских систем, обладающих свойством сверхтекучести, было высказано в работах $[32,33]$. Вопрос о релятивистском обобщении нерелятивистской теории сверхтекучести, связанный с проблематикой релятивистской астрофизики и квантовой теории поля с нарушенными симметриями, начал разрабатываться сравнительно недавно [34-41]. Другими возможными областями применения релятивистской теории сверхтекучести могут служить космология и физика элементарных части [42-45]. Так, в работах [38-41] рассматривалась релятивистская гидродинамика сверхтекучей жидкости со скалярным параметром порядка в релятивистской форме.

В данной работе получены уравнения релятивистской ферми-жидкости с тензорным параметром порядка в релятивистски-инвариантной форме. Кроме того, решены следующие задачи: введены дополнительные термодинамические параметры в терминах параметра порядка; получен второй закон термодинамики и найдены плотности потоков аддитивных интегралов движения в терминах плотности термодинамического потенциала в состоянии локального равновесия; вьведены уравнения идеальной гидродинамики сверхтекучей ферми-жидкости с триплетным спариванием без использования конкретной динамической симметрии функционала энергии; получен спектр собственных колебаний; рассмотрены случаи галилеевой и релятивистски-инвариантной физических систем.

\section{2. ФУНКЦИОНАЛ ЭНЕРГИИ И ЕГО СВОЙСТВА СИММЕТРИИ}

Описание физических процессов в квантовых системах с триплетным спариванием в ферми-жидкостном подходе основывается на задании энергии системы $E(\hat{f})=\int d^{3} x e(\mathbf{x}, \hat{f})$ как функционала статистического оператора $\hat{f}$ (одночастичной матрицы плотности) и комбинаторного выражения для энтропии

$$
\mathcal{S}(\hat{f})=-\operatorname{Sp}\{\hat{f} \ln \hat{f}+(1-\hat{f}) \ln (1-\hat{f})\} .
$$

Функционал плотности энергии системы $e(\mathbf{x}, \hat{f})$ включает в себя как кинетическую часть, так и остальные сильные взаимодействия (электростатические, обменные), которые инвариантны относительно однородных спиновых врашений и фазовых преобра- 
зований

$$
\begin{aligned}
e\left(\mathbf{x}, U_{\varphi^{0}}^{+} \hat{f} U_{\varphi^{0}}\right) & =e(\mathbf{x}, \hat{f}), & U_{\varphi^{0}} & =\exp \left(-i \varphi_{\alpha}^{0} \widehat{S}_{\alpha}\right), \\
e\left(\mathbf{x}, U_{\theta^{0}}^{+} \hat{f} U_{\theta^{0}}\right) & =e(\mathbf{x}, \hat{f}), & U_{\theta^{0}} & =\exp \left(-i \theta^{0} \widehat{N}\right),
\end{aligned}
$$

где $\varphi_{\alpha}^{0}-$ угол поворота в спиновом пространстве, $\theta^{0}$ - параметр фазового преобразования, $\widehat{S}_{\alpha}$ и $\widehat{N}$ - операторы $\alpha$-компоненты полного спина и числа частиц, соответственно. Анизотропные магнитные взаимодействия (диполь-дипольные, релятивистские) в принципе могут быть учтены в рамках теории возмушений по этим слабым взаимодействиям.

Определим операторы энергии $\hat{\varepsilon}(\hat{f})$ и плотности энергии квазичастиц $\hat{\varepsilon}(\mathbf{x}, \hat{f})$ соотношением

$$
\hat{\varepsilon}(\hat{f})=\frac{\delta E}{\delta \hat{f}}=\int d^{3} x \hat{\varepsilon}(\mathbf{x}, \hat{f}) .
$$

В силу (1) можно записать

$$
\begin{array}{cl}
U_{\varphi^{0}}^{+} \hat{\varepsilon}(\mathbf{x}, \hat{f}) U_{\varphi^{0}}=\hat{\varepsilon}\left(\mathbf{x}, U_{\varphi^{0}}^{+} \hat{f} U_{\varphi^{0}}\right), & U_{\theta^{0}}^{+} \hat{\varepsilon}(\mathbf{x}, \hat{f}) U_{\theta^{0}}=\hat{\varepsilon}\left(\mathbf{x}, U_{\theta^{0}}^{+} \hat{f} U_{\theta^{0}}\right), \\
\operatorname{Sp}\left[\widehat{S}_{\alpha}, \hat{f}\right] \hat{\varepsilon}(\mathbf{x}, \hat{f})=0, \quad \operatorname{Sp}[\widehat{N}, \hat{f}] \hat{\varepsilon}(\mathbf{x}, \hat{f})=0 .
\end{array}
$$

Функционал плотности энергии $e(\mathbf{x}, \hat{f})$, очевидно, обладает также свойством трансляционной инвариантности

$$
e\left(\mathbf{x}+\mathbf{y}, U_{\mathbf{y}}^{+} \hat{f} U_{\mathbf{y}}\right)=e(\mathbf{x}, \hat{f}), \quad U_{\mathbf{y}}=\exp \left(-i y_{k} \widehat{\mathcal{P}}_{k}\right),
$$

где $\widehat{\mathcal{P}}_{k}$ - оператор импульса, $y_{k}$ - параметр трансляции. Поэтому согласно (3) имеем

$$
U_{\mathbf{y}}^{+} \hat{\varepsilon}(\mathbf{x}-\mathbf{y}, \hat{f}) U_{\mathbf{y}}=\hat{\varepsilon}\left(\mathbf{x}, U_{\mathbf{y}}^{+} \hat{f} U_{\mathbf{y}}\right), \quad i \operatorname{Sp}\left[\widehat{\mathcal{P}}_{k}, \hat{f}\right] \hat{\varepsilon}(\mathbf{x}, \hat{f})=\nabla_{k} e(\mathbf{x}, \hat{f})
$$

Сформулируем дифференциальные законы сохранения для плотностей аддитивных интегралов движения $\zeta_{a}(\mathbf{x}, \hat{f})=\operatorname{Sp} \hat{f} \hat{\zeta}_{a}(\mathbf{x}) \quad(a=0, i, 4, \alpha)$, где операторы $\hat{\zeta}_{a}(\mathbf{x}, \hat{f})=\left\{\hat{\varepsilon}(\mathbf{x}, \hat{f}), \hat{\pi}_{i}(\mathbf{x}), \hat{n}(\mathbf{x}), \hat{s}_{\alpha}(\mathbf{x})\right\}$ могут быть записаны в виде $\hat{n}(\mathbf{x})=\hat{1} \delta(\mathbf{x}-\hat{\mathbf{x}})$, $\hat{s}_{\alpha}(\mathbf{x})=(1 / 2) \hat{\sigma}_{\alpha} \delta(\mathbf{x}-\hat{\mathbf{x}}), \quad \hat{\pi}_{i}(\mathbf{x})=(1 / 2)\left(\hat{p}_{i} \delta(\mathbf{x}-\hat{\mathbf{x}})+\delta(\mathbf{x}-\hat{\mathbf{x}}) \hat{p}_{i}\right) \quad\left(\hat{1}, \hat{\sigma}_{\alpha}-\right.$ спиновые матрицы Паули, $\delta(\mathbf{x}-\hat{\mathbf{x}})-$ операторная $\delta$-функция $)$.

Для выписанных операторов плотностей справедливы коммутационные соотношения

$$
\begin{gathered}
{\left[\hat{s}_{\alpha}(\mathbf{x}), \hat{s}_{\beta}\left(\mathbf{x}^{\prime}\right)\right]=i \varepsilon_{\alpha \beta \gamma} \hat{s}_{\gamma}(\mathbf{x}) \delta\left(\mathbf{x}-\mathbf{x}^{\prime}\right), \quad\left[\hat{n}(\mathbf{x}), \hat{n}\left(\mathbf{x}^{\prime}\right)\right]=0,} \\
{\left[\hat{\pi}_{i}(\mathbf{x}), \hat{\pi}_{j}\left(\mathbf{x}^{\prime}\right)\right]=\left(-\hat{\pi}_{j}(\mathbf{x}) \nabla_{i}+\hat{\pi}_{i}\left(\mathbf{x}^{\prime}\right) \nabla^{\prime}{ }_{j}\right) \delta\left(\mathbf{x}-\mathbf{x}^{\prime}\right),} \\
{\left[\hat{\pi}_{i}(\mathbf{x}), \hat{n}\left(\mathbf{x}^{\prime}\right)\right]=-i \hat{n}(\mathbf{x}) \nabla_{i} \delta\left(\mathbf{x}-\mathbf{x}^{\prime}\right),} \\
{\left[\hat{\pi}_{i}(\mathbf{x}), \hat{s}_{\alpha}\left(\mathbf{x}^{\prime}\right)\right]=-i \hat{s}_{\alpha}(\mathbf{x}) \nabla_{i} \delta\left(\mathbf{x}-\mathbf{x}^{\prime}\right) .}
\end{gathered}
$$

Учитывая, что статистический оператор $\hat{f}$ подчиняется уравнению движения

$$
\dot{\hat{f}}=i[\hat{f}, \hat{\varepsilon}(\hat{f})]
$$

а также свойства симметрии (2), (4) функционала энергии, найдем

$$
\dot{\zeta}_{a}(\mathbf{x}, \hat{f})=-\nabla_{k} \zeta_{a k}(\mathbf{x}, \hat{f})
$$


где $\zeta_{a k}(\mathbf{x}, \hat{f})=\operatorname{Sp} \hat{f} \hat{\zeta}_{a k}(\mathbf{x}, \hat{f})$ - плотности потоков аддитивных интегралов движения. Операторы плотности потоков $\hat{\zeta}_{a k}(\mathbf{x}, \hat{f})$ можно представить в терминах операторов плотностей $\hat{\zeta}_{a}(\mathbf{x}, \hat{f})[21]$ :

$$
\begin{gathered}
\widehat{w}_{k}(\mathbf{x}, \hat{f})=\frac{i}{2} \int d^{3} x^{\prime} x^{\prime}{ }_{k} \int_{0}^{1} d \lambda\left[\hat{\varepsilon}\left(\mathbf{x}-(1-\lambda) \mathbf{x}^{\prime}, \hat{f}\right), \hat{\varepsilon}\left(\mathbf{x}+\lambda \mathbf{x}^{\prime}\right)\right] \\
\hat{j}_{\alpha k}(\mathbf{x}, \hat{f})=i \int d^{3} x^{\prime} x^{\prime}{ }_{k} \int_{0}^{1} d \lambda\left[\hat{\varepsilon}\left(\mathbf{x}-(1-\lambda) \mathbf{x}^{\prime}, \hat{f}\right), \hat{s}_{\alpha}\left(\mathbf{x}+\lambda \mathbf{x}^{\prime}\right)\right] \\
\hat{j}_{k}(\mathbf{x}, \hat{f})=i \int d^{3} x^{\prime} x^{\prime}{ }_{k} \int_{0}^{1} d \lambda\left[\hat{\varepsilon}\left(\mathbf{x}-(1-\lambda) \mathbf{x}^{\prime}, \hat{f}\right), \hat{n}\left(\mathbf{x}+\lambda \mathbf{x}^{\prime}\right)\right]
\end{gathered}
$$

$\operatorname{Sp} \hat{f} \hat{t}_{k j}(\mathbf{x}, \hat{f})=-e(\mathbf{x}, \hat{f}) \delta_{k j}+$

$$
+i \int d^{3} x^{\prime} x^{\prime}{ }_{k} \int_{0}^{1} d \lambda \operatorname{Sp} \hat{f}\left[\hat{\varepsilon}\left(\mathbf{x}-(1-\lambda) \mathbf{x}^{\prime}, \hat{f}\right), \hat{\pi}_{j}\left(\mathbf{x}+\lambda \mathbf{x}^{\prime}\right)\right] .
$$

Последнее из соотношений (8) определено в смысле средних.

Состояние, соответствующее триплетному спариванию сверхтекучей ферми-жидкости, характеризуется параметром порядка, являюшимся симметричным спинором [5]. Оператор параметра порядка, следуя [25], выберем в виде

$$
\hat{\Delta}_{\alpha k}(\mathbf{x})=\left(\nabla_{k} \psi(\mathbf{x})\right) \hat{\sigma}_{2} \hat{\sigma}_{\alpha} \psi(\mathbf{x})-\psi(\mathbf{x}) \hat{\sigma}_{2} \hat{\sigma}_{\alpha} \nabla_{k} \psi(\mathbf{x})
$$

Этот оператор параметра порядка удовлетворяет равенствам

$$
\begin{gathered}
i\left[\hat{s}_{\alpha}(\mathbf{x}), \hat{\Delta}_{\beta i}\left(\mathbf{x}^{\prime}\right)\right]=\varepsilon_{\alpha \beta \gamma} \hat{\Delta}_{\gamma i}(\mathbf{x}) \delta\left(\mathbf{x}-\mathbf{x}^{\prime}\right)+\nabla_{i}\left(\hat{\Delta}(\mathbf{x}) \delta\left(\mathbf{x}-\mathbf{x}^{\prime}\right)\right) \delta_{\alpha \beta} \\
i\left[\hat{n}(\mathbf{x}), \hat{\Delta}_{\beta i}\left(\mathbf{x}^{\prime}\right)\right]=-2 \hat{\Delta}_{\beta i}(\mathbf{x}) \delta\left(\mathbf{x}-\mathbf{x}^{\prime}\right) \\
i\left[\hat{\pi}_{k}(\mathbf{x}), \hat{\Delta}_{\alpha i}\left(\mathbf{x}^{\prime}\right)\right]=\hat{\Delta}_{\alpha i}(\mathbf{x}) \nabla_{k} \delta\left(\mathbf{x}-\mathbf{x}^{\prime}\right)+\nabla_{i}\left(\hat{\Delta}_{\alpha k}(\mathbf{x}) \delta\left(\mathbf{x}-\mathbf{x}^{\prime}\right)\right)
\end{gathered}
$$

где $\hat{\Delta} \equiv(1 / 2) i \psi(\mathbf{x}) \hat{\sigma}_{2} \psi(\mathbf{x})$ - оператор параметра порядка синглетного спаривания сверхтекучей ферми-жидкости. В соответствии с этим определением имеем

$$
\begin{gathered}
i\left[\hat{s}_{\alpha}(\mathbf{x}), \hat{\Delta}\left(\mathbf{x}^{\prime}\right)\right]=0, \quad i\left[\hat{n}(\mathbf{x}), \hat{\Delta}\left(\mathbf{x}^{\prime}\right)\right]=-2 \hat{\Delta}(\mathbf{x}) \delta\left(\mathbf{x}-\mathbf{x}^{\prime}\right) \\
i\left[\hat{\pi}_{k}(\mathbf{x}), \hat{\Delta}\left(\mathbf{x}^{\prime}\right)\right]=\hat{\Delta}(\mathbf{x}) \nabla_{k} \delta\left(\mathbf{x}-\mathbf{x}^{\prime}\right)
\end{gathered}
$$

При локальных спиновых $U_{\varphi} \equiv \exp \left(-i \int d^{3} x \varphi_{\alpha}(\mathbf{x}) \hat{s}_{\alpha}(\mathbf{x})\right)$ и фазовых $U_{\theta} \equiv$ $\exp \left(-i \int d^{3} x \theta(\mathbf{x}) \hat{n}(\mathbf{x})\right)$ преобразованиях в силу соотношений $(2),(4),(5)$ операторы $\hat{\zeta}_{a}(\mathbf{x}, \hat{f})$ преобразуются по формулам

$$
\begin{gathered}
U_{\varphi}^{+} \hat{s}_{\alpha}(\mathbf{x}) U_{\varphi}=a_{\alpha \beta}(\varphi) \hat{s}_{\beta}(\mathbf{x}), \quad U_{\theta}^{+} \hat{s}_{\alpha}(\mathbf{x}) U_{\theta}=\hat{s}_{\alpha}(\mathbf{x}), \\
U_{\varphi}^{+} \hat{n}(\mathbf{x}) U_{\varphi}=\hat{n}(\mathbf{x}), \quad U_{\theta}^{+} \hat{n}(\mathbf{x}) U_{\theta}=\hat{n}(\mathbf{x}), \\
U_{\varphi}^{+} \hat{\pi}_{i}(\mathbf{x}) U_{\varphi}=\hat{\pi}_{i}(\mathbf{x})+\underline{\omega}_{\alpha i}(\mathbf{x}) \hat{s}_{\alpha}(\mathbf{x}), \\
U_{\theta}^{+} \hat{\pi}_{i}(\mathbf{x}) U_{\theta}=\hat{\pi}_{i}(\mathbf{x})+\nabla_{i} \theta(\mathbf{x}) \hat{n}(\mathbf{x}), \\
U_{\theta}^{+} \hat{\varepsilon}(\mathbf{x}) U_{\theta}=\hat{\varepsilon}\left(\mathbf{x}, \nabla_{i} \theta(\mathbf{x}), \hat{f}\right), \quad U_{\varphi}^{+} \hat{\varepsilon}(\mathbf{x}) U_{\varphi}=\hat{\varepsilon}\left(\mathbf{x}, \underline{\omega}_{i}(\mathbf{x}), \hat{f}\right) .
\end{gathered}
$$


Здесь $\varphi_{\alpha}(\mathbf{x})$ - локальные углы поворота, $\theta(\mathbf{x})$ - локальный параметр фазовых преобразований. Ортогональная матрица поворота $a_{\alpha \beta}(\varphi)$ связана с локальным углом поворота $\varphi_{\alpha}$ соотношением

$$
a_{\alpha \beta}(\varphi)=(\exp (-\varepsilon \varphi))_{\alpha \beta},
$$

где $(\varepsilon \varphi)_{\alpha \beta}=\varepsilon_{\alpha \beta \gamma} \varphi_{\gamma}$. В терминах матрицы поворота правая форма Картана $\underline{\omega}_{\alpha k}[46]$ определяется равенством

$$
\underline{\omega}_{\alpha k}(a)=\frac{1}{2} \varepsilon_{\alpha \beta \gamma}\left(a \nabla_{k} \tilde{a}\right)_{\gamma \beta}
$$

и удовлетворяет тождеству Маурера-Картана

$$
\nabla_{k} \underline{\omega}_{\alpha i}-\nabla_{i} \underline{\omega}_{\alpha k}=\left[\underline{\omega}_{k} \times \underline{\omega}_{i}\right]_{\alpha}
$$

При локальных фазовых преобразованиях и спиновых врашениях операторы параметров порядка синглетного и триплетного спариваний преобразуются следующим образом:

$$
\begin{gathered}
U_{\varphi}^{+} \hat{\Delta}(\mathbf{x}) U_{\varphi}=\hat{\Delta}(\mathbf{x}), \quad U_{\theta}^{+} \hat{\Delta}(\mathbf{x}) U_{\theta}=\hat{\Delta}(\mathbf{x}) \exp (-2 i \theta(\mathbf{x})) \\
U_{\varphi}^{+} \hat{\Delta}_{\alpha i}(\mathbf{x}) U_{\varphi}=a_{\alpha \beta}(\mathbf{x}) \hat{\Delta}_{\beta i}(\mathbf{x})-\underline{\omega}_{\alpha i}(\mathbf{x}) \hat{\Delta}(\mathbf{x}) \\
U_{\theta}^{+} \hat{\Delta}_{\alpha i}(\mathbf{x}) U_{\theta}=\hat{\Delta}_{\alpha i}(\mathbf{x}) \exp (-2 i \theta(\mathbf{x})) .
\end{gathered}
$$

Формулы (10), (11), (12) и (16) будут нами использованы ниже при нахождении плотностей потоков аддитивных интегралов движения и построении второго начала термодинамики.

\section{3. ОРТОГОНАЛЬНАЯ МАТРИЦА ПОВОРОТА И СВЕРХТЕКУЧАЯ ФАЗА КАК ФУНКЦИОНАЛЫ ПРОИЗВОЛЬНОГО СТАТИСТИЧЕСКОГО ОПЕРАТОРА}

В данном разделе введем ортогональную матрицу поворота и сверхтекучую фазу как функционалы неравновесного статистического оператора $\hat{f}$. Ортогональная матрица в неявном виде может быть задана выражениями

$$
\mathbf{e}_{1} a(\mathbf{x}, \hat{f}) \mathbf{u}(\mathbf{x}, \hat{f})=0, \quad \mathbf{e}_{1} a(\mathbf{x}, \hat{f}) \mathbf{v}(\mathbf{x}, \hat{f})=0, \quad \mathbf{e}_{2} a(\mathbf{x}, \hat{f}) \mathbf{v}(\mathbf{x}, \hat{f})=0 .
$$

Здесь $\mathbf{e}_{1}, \mathbf{e}_{2}, \mathbf{e}_{1} \times \mathbf{e}_{2} \quad\left(\mathbf{e}_{1}^{2}=\mathbf{e}_{2}^{2}=1, \mathbf{e}_{1} \mathbf{e}_{2}=0\right)$ - некоторый фиксированный репер, не зависящий от статистического оператора. Спиновые векторы $u_{\alpha}, v_{\alpha}$ определяются формулами

$$
\begin{gathered}
u_{\alpha}(\mathbf{x})=\operatorname{Sp} \hat{f} \hat{u}_{\alpha}(\mathbf{x}), \quad v_{\alpha}(\mathbf{x})=\operatorname{Sp} \hat{f} \hat{v}_{\alpha}(\mathbf{x}) \\
\hat{u}_{\alpha}(\mathbf{x})=i \nu_{i}\left(\hat{\Delta}_{\alpha i}(\mathbf{x}) \hat{\Delta}^{+}(\mathbf{x})-\hat{\Delta}(\mathbf{x}) \hat{\Delta}_{\alpha i}^{+}(\mathbf{x})\right) \\
\hat{v}_{\alpha}(\mathbf{x})=i \nu_{i}\left(\hat{\Delta}^{+}(\mathbf{x}) \hat{\Delta}_{\alpha i}(\mathbf{x})-\hat{\Delta}_{\alpha i}^{+}(\mathbf{x}) \hat{\Delta}(\mathbf{x})\right)
\end{gathered}
$$

где $\nu_{i}$ - единичный пространственный вектор. Легко убедиться, что при спиновых врашениях операторы (18) вследствие трансформационных свойств (16) изменяются как спиновые векторы

$$
U_{\varphi}^{+} \hat{v}_{\alpha}(\mathbf{x}) U_{\varphi}=a_{\alpha \beta}(\mathbf{x}) \hat{v}_{\beta}(\mathbf{x}), \quad U_{\varphi}^{+} \hat{u}_{\alpha}(\mathbf{x}) U_{\varphi}=a_{\alpha \beta}(\mathbf{x}) u_{\beta}(\mathbf{x}) .
$$


Из определений (18) следует, что эти векторы инвариантны относительно фазовых преобразований

$$
U_{\theta}^{+} \hat{u}_{\alpha}(\mathbf{x}) U_{\theta}=\hat{u}_{\alpha}(\mathbf{x}), \quad U_{\theta}^{+} \hat{v}_{\alpha}(\mathbf{x}) U_{\theta}=\hat{v}_{\alpha}(\mathbf{x})
$$

В силу (16), (17) справедливы формулы

$$
\begin{aligned}
a\left(\mathbf{x}, U_{\varphi}^{+} \hat{f} U_{\varphi}\right)= & a(\mathbf{x}, \hat{f}) a^{\prime}(\mathbf{x}), \quad a^{\prime}(\mathbf{x})=\exp \left(-\varepsilon \varphi^{\prime}(\mathbf{x})\right) \\
& a\left(\mathbf{x}, U_{\theta}^{+} \hat{f} U_{\theta}\right)=a(\mathbf{x}, \hat{f}) .
\end{aligned}
$$

Рассмотрим вариацию ортогональной матрицы поворота $a(\mathbf{x}, \hat{f})$, связанную с вариацией статистического оператора $\delta \hat{f}$,

$$
\delta a_{\alpha \beta}(\mathbf{x}, \hat{f})=a_{\alpha \beta}(\mathbf{x}, \hat{f}+\delta \hat{f})-a_{\alpha \beta}(\mathbf{x}, \hat{f})=a_{\alpha \gamma}(\mathbf{x}, \hat{f}) \chi_{\gamma \beta}(\mathbf{x}, \hat{f}, \delta \hat{f}),
$$

где $\chi_{\gamma \beta}(\mathbf{x}, \hat{f}, \delta \hat{f})=\operatorname{Sp} \delta \hat{f} \hat{\chi}_{\gamma \beta}(\mathbf{x}, \hat{f}), \hat{\chi}_{\gamma \beta}(\mathbf{x}, \hat{f})=-\hat{\chi}_{\beta \gamma}(\mathbf{x}, \hat{f})$.

Если теперь ввести дуальную величину $\hat{\chi}_{\gamma}(\mathbf{x}, \hat{f})=(1 / 2) \varepsilon_{\alpha \beta \gamma} \hat{\chi}_{\alpha \beta}(\mathbf{x}, \hat{f})$, то вариацию (22) можно записать в виде

$$
\delta a_{\alpha \beta}(\mathbf{x}, \hat{f})=a_{\alpha \gamma}(\mathbf{x}, \hat{f}) \varepsilon_{\gamma \beta \lambda} \operatorname{Sp} \delta \hat{f} \hat{\chi}_{\lambda}(\mathbf{x}, \hat{f}) \equiv \operatorname{Sp} \delta \hat{f} \hat{a}_{\alpha \beta}(\mathbf{x}, \hat{f})
$$

Как видно из (23), оператор ортогональной матрицы $\hat{a}_{\alpha \beta}$ связан с оператором $\hat{\chi}_{\gamma}$ соотношением

$$
\hat{a}_{\alpha \beta}(\mathbf{x}, \hat{f})=\hat{a}_{\alpha \gamma}(\mathbf{x}, \hat{f}) \varepsilon_{\gamma \beta \lambda} \hat{\chi}_{\lambda}(\mathbf{x}, \hat{f}) .
$$

В силу (21) введенный оператор $\hat{a}_{\alpha \beta}$ удовлетворяет соотношениям

$$
i \operatorname{Sp} \hat{f}\left[\hat{s}_{\alpha}\left(\mathbf{x}^{\prime}\right), \hat{a}_{\beta \gamma}(\mathbf{x}, \hat{f})\right]=\varepsilon_{\alpha \rho \gamma} a_{\beta \rho}(\mathbf{x}) \delta\left(\mathbf{x}-\mathbf{x}^{\prime}\right), \quad i \operatorname{Sp} \hat{f}\left[\hat{n}\left(\mathbf{x}^{\prime}\right), \hat{a}_{\alpha \beta}(\mathbf{x}, \hat{f})\right]=0 .
$$

При преобразованиях произвольных деформаций, задаваемых унитарным преобразованием $U_{\xi} \equiv \exp \left(-i \int d^{3} x \xi_{i}(\mathbf{x}) \hat{\pi}_{i}(\mathbf{x})\right)$, ортогональная матрица меняется следующим образом:

$$
a\left(\mathbf{x}, U_{\xi}^{+} \hat{f} U_{\xi}\right)=a\left(\mathbf{x}^{\prime}(\mathbf{x}), \hat{f}\right) .
$$

В формуле $(26) \mathbf{x}^{\prime}=\mathbf{x}^{\prime}(\mathbf{x}, \xi)$ является сложной функцией от координаты $\mathbf{x}$. В случае бесконечно малых преобразований деформации эта связь имеет вид $\delta x_{i}^{\prime}=\delta x_{i}-\delta \xi_{i}$. Учитывая формулы (10), (11), (26), приходим к соотношению

$$
i \operatorname{Sp} \hat{f}\left[\hat{\pi}_{i}(\mathbf{x}), \hat{a}_{\alpha \beta}\left(\mathbf{x}^{\prime}, \hat{f}\right)\right]=\nabla_{i} a_{\alpha \beta}(\mathbf{x}) \delta\left(\mathbf{x}-\mathbf{x}^{\prime}\right) .
$$

Варьируя (17) и используя свойства $(25)-(27)$ оператора $\hat{a}_{\alpha \beta}$, найдем его явный вид в терминах оператора параметра порядка

$$
\hat{a}_{\alpha \beta}=\frac{1}{\kappa^{2}}\left\{(\hat{\mathbf{v}} \boldsymbol{\kappa}) u_{\rho}-(\hat{\mathbf{u}} \boldsymbol{\kappa}) v_{\rho}+\frac{\kappa_{\rho}}{v^{2}}\left[(\mathbf{u v})\left(\mathbf{v} \hat{\mathbf{v}}-(\mathbf{u} \hat{\mathbf{v}}) v^{2}\right)\right]\right\} a_{\alpha \gamma} \varepsilon_{\gamma \beta \rho},
$$

где $\boldsymbol{\kappa} \equiv \mathbf{u} \times \mathbf{v}$.

Сверхтекучую фазу $\theta(\mathbf{x})$ как функционал статистического оператора определим формулой

$$
\theta(\mathbf{x})=\frac{1}{4} \operatorname{Im} \ln \left(\operatorname{Sp} \hat{f} \hat{\Delta}_{\alpha k}(\mathbf{x})\right)^{2} .
$$


В соответствии с (16), (29) получим закон преобразования сверхтекучей фазы при локальных спиновых врашениях и фазовых преобразованиях

$$
\theta\left(\mathbf{x}, U_{\varphi}^{+} \hat{f} U_{\varphi}\right)=\theta(\mathbf{x}, \hat{f}), \quad \theta\left(\mathbf{x}, U_{\vartheta}^{+} \hat{f} U_{\vartheta}\right)=\theta(\mathbf{x}, \hat{f})+\vartheta(\mathbf{x})
$$

Рассмотрим вариацию сверхтекучей фазы, связанную с вариацией статистического оператора,

$$
\delta \theta(\mathbf{x}, \hat{f})=\theta(\mathbf{x}, \hat{f}+\delta \hat{f})-\theta(\mathbf{x}, \hat{f})=\operatorname{Sp} \delta \hat{f} \hat{\vartheta}(\mathbf{x}) .
$$

Здесь согласно определению (29) и формуле (31) оператор сверхтекучей фазы $\hat{\vartheta}(\mathbf{x})$ в терминах оператора параметра порядка имеет следующий вид:

$$
\hat{\vartheta}=i\left\{\Delta_{\alpha i} \hat{\Delta}_{\alpha i} \Delta_{\beta j}^{-2}-\Delta_{\alpha i}^{*} \hat{\Delta}_{\alpha i}^{+} \Delta_{\beta j}^{*}{ }^{-2}\right\}
$$

Поступая так же, как и при получении свойств оператора ортогональной матрицы, найдем соотношения, которым удовлетворяет оператор сверхтекучей фазы,

$$
\begin{gathered}
i \operatorname{Sp} \hat{f}\left[\hat{s}_{\alpha}\left(\mathbf{x}^{\prime}\right), \hat{\vartheta}(\mathbf{x}, \hat{f})\right]=0, \quad i \operatorname{Sp} \hat{f}\left[\hat{n}\left(\mathbf{x}^{\prime}\right), \hat{\vartheta}(\mathbf{x}, \hat{f})\right]=\delta\left(\mathbf{x}-\mathbf{x}^{\prime}\right), \\
i \operatorname{Sp} \hat{f}\left[\hat{\pi}_{k}\left(\mathbf{x}^{\prime}\right), \hat{\vartheta}(\mathbf{x}, \hat{f})\right]=\nabla_{k} \theta(\mathbf{x}, \hat{f}) \delta\left(\mathbf{x}-\mathbf{x}^{\prime}\right) .
\end{gathered}
$$

Явные выражения для операторов $\hat{a}(\mathbf{x})(28), \hat{\vartheta}(\mathbf{x})(32)$ и их свойства $(25),(27),(33)$ ниже будут использованы при получении динамических уравнений для ортогональной матрицы и сверхтекучей фазы.

Исходя из найденного явного вида оператора ортогональной матрицы (28) получим выражение для левой формы Картана $\omega_{\alpha k}$ в терминах параметра порядка

$$
\omega_{\alpha k} \equiv a_{\beta \alpha} \underline{\omega}_{\beta k}=z_{\alpha k}+d_{\alpha} q_{k},
$$

где $z_{\alpha k}=\varepsilon_{\alpha \gamma \beta} d_{\beta} \nabla_{k} d_{\gamma}-$ поперечная по отношению к вектору $\mathbf{d}$ часть формы Картана, связанная с параметром порядка соотношениями

$$
d_{\alpha}=\kappa_{\alpha} /|\kappa|, \quad \kappa=\mathbf{u} \times \mathbf{v}, \quad d^{2}=1 .
$$

Из тождества Маурера-Картана (14) и вида левой формы Картана $\omega_{\alpha k}(34)$ приходим к равенствам

$$
\nabla_{i} q_{k}-\nabla_{k} q_{i}=\varepsilon_{\alpha \beta \gamma} d_{\alpha} \nabla_{i} d_{\beta} \nabla_{k} d_{\gamma}, \quad \nabla_{k} z_{\alpha i}-\nabla_{i} z_{\alpha k}=\varepsilon_{\alpha \beta \gamma} z_{\beta i} z_{\gamma k} .
$$

Первое из них представляет собой тождество Мермина-Хо [47]. Второе определяет тождество для поперечных составляюших формы Картана относительно направления вектора d, имеюшего смысл вектора магнитной анизотропии.

\section{4. КВАЗИСРЕДНИЕ В ФЕРМИ-ЖИДКОСТНОМ ПОДХОДЕ}

Равновесный статистический оператор нормального состояния ферми-жидкости $\hat{f}$ находится из требования максимума энтропии при фиксированных значениях аддитивных интегралов движения $\gamma_{a}$. Из условия экстремума для термодинамического потенциала $\Omega$ (варьирование производится по статистическому оператору $\hat{f}$ )

$$
\delta \Omega(\hat{f}, Y)=0, \quad \Omega(\hat{f}, Y)=-\mathcal{S}(\hat{f})+Y_{a} \gamma_{a}
$$


следует уравнение самосогласования для равновесного одночастичного оператора

$$
\hat{f}=\left[\exp \left(Y_{a} \hat{\gamma}_{a}(\hat{f})\right)+1\right]^{-1} .
$$

Здесь $Y_{a}=\left\{Y_{0}, Y_{\alpha}, Y_{i}, Y_{4}\right\}$ - множители Лагранжа, соответствующие величинам $\gamma_{a}=$ $\left(E(\hat{f}), S_{\alpha}, \mathcal{P}_{k}, N\right)$.

Равновесное состояние нормальной ферми-жидкости характеризуется только термодинамическими параметрами $Y_{a}=\left(Y_{0}, Y_{i}, Y_{4}, Y_{\alpha}\right)$. Состояние, описываемое статистическим оператором (36), обладает в соответствии с $(2),(4)$ свойствами симметрии

$$
\left[\hat{f}, \widehat{\mathcal{P}}_{i}\right]=0, \quad\left[\hat{f}, \widehat{\Sigma}_{\alpha}\right]=0, \quad[\hat{f}, \hat{\varepsilon}(\hat{f})]=0, \quad[\hat{f}, \widehat{N}]=0, \quad\left[\hat{f}, \hat{L}_{i}\right]=0 .
$$

Здесь $\widehat{\Sigma}_{\alpha}=\widehat{S}_{\alpha}-i \varepsilon_{\alpha \beta \gamma} Y_{\beta} \partial / \partial Y_{\gamma}, \hat{L}_{i}=\hat{\mathcal{L}}_{i}-i \varepsilon_{i k l} Y_{k} \partial / \partial Y_{l}$ - обобщенные операторы спина и орбитального момента, а $\hat{\mathcal{L}}_{i}=\int d^{3} x \varepsilon_{i j k} x_{j} \hat{\pi}_{k}(\mathbf{x})$ - орбитальный момент. Легко видеть, что для состояния (36) справедлив второй закон термодинамики

$$
d \omega=\zeta_{a} d Y_{a}, \quad \omega=\lim _{V \rightarrow \infty} \Omega / V=\omega(Y) .
$$

В конденсированной среде со спонтанно нарушенной симметрией существуют различные возможности нарушения трансляционной инвариантности, под которой понимаем следуюшее соотношение: $\left[\hat{f}, \widehat{\mathcal{P}}_{i}\right]=0$. Нарушение этого соотношения может произойти как вследствие нарушения фазовой инвариантности (сверхтекучий импульс не равен нулю) или инвариантности относительно врашения спинов (вектор магнитной спирали отличен от нуля), так и вследствие нарушения симметрии в конфигурационном пространстве (состояние типа холестерика с вектором немагнитной спирали, не равным нулю). Мы рассмотрим возможности, связанные с появлением состояния равновесия с отличными от нуля сверхтекучим импульсом и вектором магнитной спирали.

Для описания В-фазы сверхтекучей ферми-жидкости модифицируем определение равновесного статистического оператора. С этой целью в соответствии с концепией квазисредних определим равновесное среднее равенством

$$
\begin{aligned}
a(\mathbf{x}, \hat{f}) & =\operatorname{Sp} \hat{f} \hat{a}(\mathbf{x})=\lim _{\nu \rightarrow 0} \lim _{V \rightarrow \infty} \operatorname{Sp} \hat{f}_{\nu} \hat{a}(\mathbf{x}), \\
\hat{f}_{\nu} & =\left[\exp \left(Y_{a} \hat{\gamma}_{a}+\nu Y_{0} \widehat{G}\right)+1\right]^{-1}
\end{aligned}
$$

где $\hat{a}(\mathbf{x})$ - произвольный квазилокальный оператор, $\widehat{G}$ - источник, нарушаюший соотношения симметрии (37) и представляющий собой линейный функционал операторов параметров порядка $\hat{\Delta}, \hat{\Delta}_{\alpha k}$ :

$$
\widehat{G}=\int d^{3} x\left\{g_{\alpha k}(\mathbf{x}, t) \hat{\Delta}_{\alpha k}(\mathbf{x})+g(\mathbf{x}, t) \hat{\Delta}(\mathbf{x})+\text { э. c. }\right\} .
$$

Конкретный вид функций $g(\mathbf{x}, t), g_{\alpha k}(\mathbf{x}, t)$ определяется свойствами симметрии состояния равновесия, которые для В-фазы сверхтекучей ферми-жидкости имеют вид

$$
\begin{gathered}
{\left[\hat{f}, \widehat{\mathcal{P}}_{k}-q_{k} n_{\alpha} \widehat{\Sigma}_{\alpha}-p_{k} \widehat{N}\right]=0, \quad\left[\hat{f}, Y_{0} \hat{\varepsilon}(\hat{f})+Y_{0} \omega_{\alpha 0} \widehat{\Sigma}_{\alpha}+Y_{0} p_{0} \widehat{N}\right]=0,} \\
{\left[\hat{f}, \hat{L}_{k}^{\prime}+\hat{a}_{k \alpha}^{\prime} \widehat{\Sigma}_{\alpha}\right]=0, \quad \hat{L}_{i}^{\prime}=\hat{L}_{i}-i \varepsilon_{i k l}\left(p_{k} \partial / \partial p_{l}+q_{k} \partial / \partial q_{l}\right),} \\
\omega_{\alpha 0}=Y_{0}^{-1}\left(Y_{\alpha}+Y_{k} q_{k} n_{\alpha}\right), \quad p_{0}=Y_{0}^{-1}\left(Y_{4}+Y_{k} p_{k}\right) .
\end{gathered}
$$


Здесь $q_{k}$ - вектор магнитной спирали, $p_{k}$ - сверхтекучий импульс. Единичный вектор $n_{\alpha}$ находится из условия непротиворечивости соотношений (40) и представим в виде $n_{\alpha}=Y_{\alpha} /|Y|$. Первое из соотношений (40) определяет условие пространственной однородности. Второе- -условие стационарности сверхтекучей жидкости. Третье определяет взаимную ориентацию спиновой и орбитальной подсистем. Условия пространственной симметрии играют сушественную роль при построении второго начала термодинамики и получении уравнений идеальной гидродинамики. Ранее подобного рода соотношения формулировались для сверхтекучих систем с синглетным спариванием [25] и для многоподрешеточных магнетиков [31]. Выписанные нами свойства симметрии являются обобщением условий симметрии на рассматриваемые квантовые жидкости с триплетным спариванием.

Согласно (6), (40) получим явньй вид функций $g, g_{\alpha k}$

$$
\begin{gathered}
g(\mathbf{x}, t)=\exp (-2 i \theta(\mathbf{x})), \quad g_{\alpha k}(\mathbf{x}, t)=a_{k \beta}^{\prime} a_{\beta \alpha}(\varphi(\mathbf{x}, t)) \exp (-2 i \theta(\mathbf{x})), \\
\varphi_{\alpha}(\mathbf{x}, t)=n_{\alpha}\left(\mathbf{q} \mathbf{x}+q_{0} t+\varphi_{0}\right), \quad \theta(\mathbf{x}, t)=\theta_{0}+\mathbf{p x}+p_{0} t,
\end{gathered}
$$

где $q_{0} \equiv n_{\alpha} \omega_{\alpha 0}, a_{k \alpha}^{\prime}$ - матрица однородного поворота спиновой подсистемы относительно орбитальной. Отметим, что в соответствии с определениями (14) правая форма Картана для матришы поворота $a(\mathbf{x}, t)$ равна

$$
\underline{\omega}_{\alpha k}=q_{k} \underline{n}_{\alpha}, \quad \underline{n}_{\alpha}=a_{\alpha \beta}\left(\varphi_{0}\right) n_{\beta}
$$

и не зависит от координат и времени в состоянии полного равновесия. Из условий симметрии (38) следует, что равновесные значения плотности спина $s_{\alpha}(\mathbf{x}, t)$ зависят от координат и времени посредством матришы поворота, т.е. $s_{\alpha}(\mathbf{x}, t)=a_{\beta \alpha}(\mathbf{x}, t) \underline{s}_{\beta}$. Сверхтекучий импульс и средние остальных аддитивных интегралов движения не зависят от координат и времени в состоянии равновесия. Таким образом, состояние равновесия В-фазы сверхтекучей ферми-жидкости характеризуется термодинамическими параметрами $Y_{a}$, вектором спирали $\mathbf{q}$, сверхтекучим импульсом $\mathbf{p}$, углами поворота $\varphi_{\alpha}^{0}$ и сверхтекучей фазой $\theta^{0}$.

\section{5. ТЕРМОДИНАМИКА И ПОТОКИ В ЛОКАЛЬНО-РАВНОВЕСНОМ СОСТОЯНИИ}

Для конденсированных сред с нарушенной неабелевой группой симметрии не все равновесные плотности потоков представимы в терминах равновесного термодинамического потенциала $[31,48]$. Причина этого связана с тем, что некоторые плотности потоков в состоянии равновесия обрашаются в нуль $[31,48]$. Поэтому при формулировке термодинамики и нахождении плотностей потоков адлитивных интегралов движения целесообразно исходить из локально-равновесного состояния, статистический оператор которого определяется равенством

$$
\begin{aligned}
& \hat{f}\{Y(\mathbf{x}), a(\mathbf{x}), \theta(\mathbf{x})\}=\left[\exp \int d^{3} x\left(Y_{a}(\mathbf{x}) \hat{\zeta}_{a}(\mathbf{x}, \hat{f})\right)+\right. \\
& \left.\quad+\nu Y_{0}(\mathbf{x}) U_{\theta}^{+} U_{\varphi}^{+}\left(g(\mathbf{x}) \hat{\Delta}(\mathbf{x})+\mathbf{g}_{\alpha}(\mathbf{x}) \hat{\Delta}_{\alpha}(\mathbf{x})+\text { э. c. }\right) U_{\varphi} U_{\theta}+1\right]^{-1}
\end{aligned}
$$

6 Теоретическая и математическая физика, т. 113, № 2, 1997 г. 
где $g(\mathbf{x}), \mathbf{g}_{\alpha}(\mathbf{x})$ - некоторые $c$-числовые функции. Для таких состояний плотности потоков в общем случае не обрашаются в нуль и, как будет показано ниже, могут быть представлены в терминах термодинамического потенциала. В локально-равновесном состоянии термодинамические параметры $Y(\mathbf{x})$, сверхтекучая фаза $\theta(\mathbf{x})$ и ортогональная матрица $a(\mathbf{x})$ являются произвольными функциями координат. В состоянии полного равновесия $Y(\mathbf{x})=Y$ и структура сверхтекучей фазы $\theta(\mathbf{x})$ и ортогональной матрищы $a_{\alpha \beta}(\mathbf{x})$ определяются формулой (41). Рассматривая локально-равновесное состояние, целесообразно перейти от оператора (43) к новому оператору

$$
\begin{aligned}
\underline{\hat{f}}\{Y, a, \theta\}= & U_{\theta} U_{\varphi} \hat{f}\{Y, a, \theta\} U_{\varphi}^{+} U_{\theta}^{+}= \\
= & {\left[\operatorname { e x p } \int d ^ { 3 } x \left(Y_{a}(\mathbf{x}) U_{\theta} U_{\varphi} \hat{\zeta}_{a}(\mathbf{x}) U_{\varphi}^{+} U_{\theta}^{+}+\right.\right.} \\
& \left.\left.+\nu Y_{0}(\mathbf{x})\left(g(\mathbf{x}) \hat{\Delta}(\mathbf{x})+\mathbf{g}_{\alpha}(\mathbf{x}) \hat{\Delta}_{\alpha}(\mathbf{x})+\text { э. с. }\right)\right)+1\right]^{-1}
\end{aligned}
$$

Тогда для локально-равновесного термодинамического потенциала имеет место равенство

$$
\begin{aligned}
\Omega(\hat{f}(Y, a, \theta) Y) & =-\mathcal{S}(\hat{f})+\int d^{3} x Y_{a}(\mathbf{x}) \operatorname{Sp} \hat{f} \hat{\zeta}_{a}(\mathbf{x})= \\
& =-\mathcal{S}(\underline{\hat{f}})+\int d^{3} x Y_{a}(\mathbf{x}) \operatorname{Sp} \underline{\hat{f}} \hat{\zeta}_{a}(\mathbf{x})
\end{aligned}
$$

Здесь $Y_{a}(\mathbf{x})$ - множители Лагранжа, соответствующие величинам $\zeta_{a}(\mathbf{x})=\operatorname{Sp} \hat{f} \hat{\zeta}_{a}(\mathbf{x})$. Из (12), (44), (45) следует, что локально-равновесный термодинамический потенциал $\Omega$ является функционалом термодинамических параметров $\underline{Y}_{a}=\left\{Y_{0}, \underline{Y}_{\alpha}=\right.$ $\left.a_{\alpha \beta} Y_{\beta}, Y_{i}, Y_{4}\right\}$, правой формы Картана $\underline{\omega}_{\alpha k}\left(\mathbf{x}^{\prime}\right)$ и сверхтекучего импульса $\mathbf{p}=\nabla \theta$ :

$$
\Omega=\Omega\left(\underline{Y}\left(\mathbf{x}^{\prime}\right), \underline{\omega}_{k}\left(\mathbf{x}^{\prime}\right), \mathbf{p}\left(\mathbf{x}^{\prime}\right)\right)=\int d^{3} x \omega\left(\mathbf{x}, \underline{Y}\left(\mathbf{x}^{\prime}\right), \underline{\omega}_{k}\left(\mathbf{x}^{\prime}\right), \theta\left(\mathbf{x}^{\prime}\right)\right) .
$$

Здесь $\omega$ - плотность термодинамического потенциала. Инфинитезимальными характеристиками унитарных преобразований $U_{\varphi}, U_{\theta}$ являются операторы $U_{\varphi}^{+} \delta U_{\varphi}, U_{\theta}^{+} \delta U_{\theta}$, которые можно записать в следуюшем виде:

$$
\begin{gathered}
U_{\varphi}^{+} \delta U_{\varphi}=i \int d^{3} x \delta R_{\gamma}(\mathbf{x}) \hat{s}_{\gamma}(\mathbf{x}), \quad \delta R_{\gamma}=\frac{1}{2} \varepsilon_{\alpha \beta \gamma}(a \delta \tilde{a})_{\alpha \beta} \\
U_{\theta}^{+} \delta U_{\theta}=i \int d^{3} x \delta \theta(\mathbf{x}) \hat{n}(\mathbf{x}) .
\end{gathered}
$$

Здесь $\delta U_{\varphi}$ - вариация оператора $U_{\varphi}$, обусловленная вариацией ортогональной матрицы поворота $\delta a_{\alpha \beta}$, а $\delta U_{\theta}$ - вариация оператора $U_{\theta}$, обусловленная вариацией фазы $\delta \theta$.

Варьируя потенциал $\Omega(45)$ по термодинамическим параметрам, получим второе начало термодинамики для локально-равновесного состояния

$$
\delta \omega=\underline{\zeta}_{a} \delta \underline{Y}_{a}+\left(Y_{0} \underline{j}_{\alpha k}+Y_{k} \underline{s}_{\alpha}\right) \delta \underline{\omega}_{\alpha k}+\left(Y_{0} j_{k}+Y_{k} n\right) \delta p_{k}
$$


где $\underline{\zeta}_{a}=\operatorname{Sp} \underline{\hat{f}} \zeta_{a}=\left\{\varepsilon, \pi_{i}, \underline{s}_{\alpha}=a_{\alpha \beta} s_{\beta}, n\right\}$

$$
\underline{j}_{\alpha k}=a_{\alpha \beta} j_{\beta k}=\frac{1}{Y_{0}} \frac{\delta \omega}{\delta \underline{\omega}_{\alpha k}}-\frac{Y_{k}}{Y_{0}} \frac{\delta \omega}{\delta \underline{Y}_{\alpha}}, \quad j_{k}=\frac{1}{Y_{0}} \frac{\delta \omega}{\delta p_{k}}-\frac{Y_{k}}{Y_{0}} \frac{\delta \omega}{\delta Y_{4}} .
$$

Найдем теперь выражение для тензора натяжений. С этой целью рассмотрим модифицированный потенциал, связанный с группой произвольных деформаций $\xi_{i}(\mathbf{x})$,

$$
\Omega(f(Y, a, \theta, \xi), Y)=-\mathcal{S}(\hat{f})+\int d^{3} x Y_{a}(\mathbf{x}) \operatorname{Sp} U_{\xi} \hat{f} U_{\xi}^{+} \hat{\zeta}_{a}(\mathbf{x}) .
$$

Его вариация может быть записана в виде

$$
\delta_{\xi} \Omega=-i \int d^{3} x d^{3} x^{\prime} \delta \xi_{i}\left(\mathbf{x}^{\prime}\right) \operatorname{Sp} \hat{f}\left[\hat{\pi}_{i}\left(\mathbf{x}^{\prime}\right), Y_{a}(\mathbf{x}) \hat{\zeta}_{a}(\mathbf{x})\right] .
$$

Используя соотношения (5), получим

$$
\begin{aligned}
\delta_{\xi} \Omega= & \int d^{3} x \delta \xi_{i}(\mathbf{x})\left\{-\nabla_{k}\left(Y_{0}(\mathbf{x}) t_{i k}(\mathbf{x})+\frac{\partial \omega Y_{k}}{\partial Y_{i}}\right)+\right. \\
& \left.+\frac{\partial \omega}{\partial p_{k}} \nabla_{i} p_{k}(\mathbf{x})+\frac{\partial \omega}{\partial \underline{\omega}_{\alpha k}} \nabla_{i} \underline{\omega}_{\alpha k}(\mathbf{x})\right\} .
\end{aligned}
$$

С другой стороны, исходя из явного вида сверхтекучего импульса $p_{k} \equiv p_{k}\left(U_{\xi} \hat{f} U_{\xi}^{+}\right)$ и формы Картана $\underline{\omega}_{\alpha k} \equiv \underline{\omega}_{\alpha k}\left(U_{\xi} \hat{f} U_{\xi}^{+}\right)$найдем их трансформационные свойства при бесконечно малых преобразованиях деформаций $\delta p_{k}=-\nabla_{k} \delta \eta_{i} p_{i}-\nabla_{i} p_{k} \delta \eta_{i}, \quad \delta \underline{\omega}_{\alpha k}=$ $-\nabla_{k} \delta \xi_{i} \underline{\omega}_{\alpha i}+\nabla_{i} \underline{\omega}_{\alpha k} \delta \xi_{i}$. Откуда, учитывая (46), получим вариацию

$$
\begin{aligned}
\delta_{\xi} \Omega & =\int d^{3} x \delta_{\xi} \omega(\mathbf{x})=\int d^{3} x\left\{\frac{\partial \omega}{\partial p_{k}} \delta_{\xi} p_{k}(\mathbf{x})+\frac{\partial \omega}{\partial \underline{\omega}_{\alpha k}} \delta_{\xi} \underline{\omega}_{\alpha k}(\mathbf{x})\right\}= \\
& =\int d^{3} x\left\{\frac{\partial \omega}{\partial p_{k}}\left(p_{i} \nabla_{k} \delta \xi_{i}+\nabla_{i} p_{k} \delta \xi_{i}\right)+\frac{\partial \omega}{\partial \underline{\omega}_{\alpha k}}\left(\underline{\omega}_{\alpha i} \nabla_{k} \delta \xi_{i}+\nabla_{i} \underline{\omega}_{\alpha k} \delta \xi_{i}\right)\right\} .
\end{aligned}
$$

Сравнивая два последних выражения для $\delta_{\xi} \Omega$, найдем тензор натяжений

$$
t_{i k}=-\frac{\partial}{\partial Y_{i}} \frac{\omega Y_{k}}{Y_{0}}+\frac{p_{i}}{Y_{0}} \frac{\partial \omega}{\partial p_{k}}+\frac{\underline{\omega}_{\alpha i}}{Y_{0}} \frac{\partial \omega}{\partial \underline{\omega}_{\alpha k}} .
$$

Чтобы получить плотность потока энергии, следует обобщить на случай локального равновесия теорему (см. [49]), связываюшую плотности потоков адлитивных интегралов движения в состоянии равновесия. С этой целью будем исходить из равенства

$$
i \operatorname{Sp} \hat{f}\left[\hat{A}_{\nu}+\widehat{B}, \hat{a}_{\nu}(\mathbf{x})+\hat{b}(\mathbf{x})\right] \equiv 0,
$$

где

$$
\begin{aligned}
\hat{A}_{\nu} & =\int d^{3} x \hat{a}_{\nu}(\mathbf{x})=\int d^{3} x Y_{0}(\mathbf{x}) \hat{\varepsilon}_{\nu}(\mathbf{x}), \\
\widehat{B} & =\int d^{3} x \hat{b}(\mathbf{x})=\int d^{3} x Y_{a^{\prime}}(\mathbf{x}) \hat{\zeta}_{a^{\prime}}(\mathbf{x}), \quad a^{\prime}=i, 4, \alpha .
\end{aligned}
$$


В соответствии с тождеством [21]

$$
\begin{gathered}
i[\hat{A}, \hat{b}(\mathbf{x})]=-i[\widehat{B}, \hat{a}(\mathbf{x})]-\nabla_{k} \hat{b}_{k}(\mathbf{x}), \\
\hat{b}_{k}(\mathbf{x})=i \int d^{3} x^{\prime} x^{\prime}{ }_{k} \int_{0}^{1} d \lambda\left[\hat{a}\left(\mathbf{x}-(1-\lambda) \mathbf{x}^{\prime}\right), \hat{b}\left(\mathbf{x}+\lambda \mathbf{x}^{\prime}\right)\right],
\end{gathered}
$$

получим, что

$$
\begin{gathered}
i \operatorname{Sp} \hat{f}\left[\hat{A}_{\nu}, \hat{a}_{\nu}(\mathbf{x})\right]=-\nabla_{k} Q_{k}^{\nu}(\mathbf{x}), \quad i \operatorname{Sp} \hat{f}[\widehat{B}, \hat{b}(\mathbf{x})]=-\nabla_{k} T_{k}(\mathbf{x}), \\
i \operatorname{Sp} \hat{f}\left[\hat{A}_{\nu}, \hat{b}(\mathbf{x})\right]+i \operatorname{Sp} \hat{f}\left[\widehat{B}, \hat{a}_{\nu}(\mathbf{x})\right]=-\nabla_{k} D_{k}^{\nu}(\mathbf{x})
\end{gathered}
$$

где

$$
\begin{aligned}
Q_{k}^{\nu}(\mathbf{x})= & \frac{i}{2} \int d^{3} x^{\prime} x^{\prime}{ }_{k} \int_{0}^{1} d \lambda Y_{0}\left(\mathbf{x}-(1-\lambda) \mathbf{x}^{\prime}\right) Y_{0}\left(\mathbf{x}+\lambda \mathbf{x}^{\prime}\right) \times \\
& \times \operatorname{Sp} \hat{f}\left[\hat{\varepsilon}\left(\mathbf{x}-(1-\lambda) \mathbf{x}^{\prime}, \hat{f}\right), \hat{\varepsilon}\left(\mathbf{x}+\lambda \mathbf{x}^{\prime}\right)\right], \\
T_{k}(\mathbf{x})= & \frac{i}{2} \int d^{3} x^{\prime} x^{\prime}{ }_{k} \int_{0}^{1} d \lambda Y_{a}\left(\mathbf{x}-(1-\lambda) \mathbf{x}^{\prime}\right) Y_{b}\left(\mathbf{x}+\lambda \mathbf{x}^{\prime}\right) \times \\
& \times \operatorname{Sp} \hat{f}\left[\hat{\zeta}_{a}\left(\mathbf{x}-(1-\lambda) \mathbf{x}^{\prime}, \hat{f}\right), \hat{\zeta}_{b}\left(\mathbf{x}+\lambda \mathbf{x}^{\prime}\right)\right], \\
D_{k}^{\nu}(\mathbf{x}, \hat{f})= & i \int d^{3} x^{\prime} x^{\prime}{ }_{k} \int_{0}^{1} d \lambda Y_{0}\left(\mathbf{x}-(1-\lambda) \mathbf{x}^{\prime}\right) Y_{b}\left(\mathbf{x}+\lambda \mathbf{x}^{\prime}\right) \times \\
& \times \operatorname{Sp} \hat{f}\left[\hat{\varepsilon}\left(\mathbf{x}-(1-\lambda) \mathbf{x}^{\prime}, \hat{f}\right), \hat{\zeta}_{b}\left(\mathbf{x}+\lambda \mathbf{x}^{\prime}\right)\right] .
\end{aligned}
$$

Пренебрегая здесь градиентами термодинамических параметров $Y_{a}$ и учитывая соотношения (8), найдем

$$
\nabla_{k}\left(Q_{\nu k}^{0}+D_{\nu k}^{0}+T_{k}^{0}\right)=0
$$

где $Q_{\nu k}^{0}=Y_{0}^{2} \omega_{\nu k}, D_{\nu k}^{0}=Y_{0} Y_{a} j_{a k}+Y_{0} Y_{k} \varepsilon_{\nu}, T_{k}^{0}=Y_{k} Y_{a} \zeta_{a}$. Отсюда следует, что вектор $Q_{k}+D_{k}+T_{k}$ не зависит от термодинамических параметров и координат, т.е. является постоянным, и в результате отсутствия какого-либо выделенного направления в пространстве этот постоянный вектор равен нулю. Следовательно, переходя к термодинамическому пределу, из выражения (50) получим

$$
\underline{Y}_{a}\left(Y_{k} \underline{\zeta}_{a}+Y_{0} \underline{\zeta}_{a k}\right)=0
$$

Это соотношение обобшает теорему о связи потоков аддитивных интегралов движения [49] на случай локально-равновесных состояний. Отсюда найдем плотность потока энергии

$$
w_{k}=-\frac{\partial}{\partial Y_{0}} \frac{\omega Y_{k}}{Y_{0}}-\frac{p_{0}}{Y_{0}} \frac{\partial \omega}{\partial p_{k}}-\frac{\underline{\omega}_{\alpha 0}}{Y_{0}} \frac{\partial \omega}{\partial \underline{\omega}_{\alpha k}} .
$$

Все найденные выражения для плотностей потоков $(47),(48),(51)$ в локально-равновесном состоянии можно записать в едином компактном виде:

$$
\underline{\zeta}_{a k}=-\frac{\partial}{\partial \underline{Y}_{a}} \frac{\omega Y_{k}}{Y_{0}}+\frac{\partial p_{0}}{\partial \underline{Y}_{a}} \frac{\partial \omega}{\partial p_{k}}+\frac{\partial \underline{\omega}_{\alpha 0}}{\partial \underline{Y}_{a}} \frac{\partial \omega}{\partial \underline{\omega}_{\alpha k}} .
$$


Если в формулах $(46),\left(47^{\prime}\right),(48),(51)$ исключить из набора термодинамических параметров форму Картана $\underline{\omega}_{\alpha k}$ и величину $\underline{Y}_{\alpha}$, то мы получим второе начало термодинамики и выражения для потоков физических величин в сверхтекучих системах с синглетным спариванием $[3,27,28]$. Если в формулах $(46),\left(47^{\prime}\right),(48),(51)$ исключить сверхтекучий импульс $p_{i}$ и параметры $Y_{4}, Y_{i}$, то получим выражения для потоков, соответствуюших многоподрешеточным магнетикам [30, 31].

Используя очевидное соотношение

$$
i \int d^{3} x^{\prime} \operatorname{Sp} \hat{f}\left[\hat{s}_{\alpha}(\mathbf{x}), Y_{a}\left(\mathbf{x}^{\prime}\right) \hat{\zeta}_{a}\left(\mathbf{x}^{\prime}\right)\right]=0
$$

придем к следуюшему равенству, отражаюшему свойства инвариантности термодинамического потенциала по отношению к спиновым врашениям:

$$
\varepsilon_{\alpha \beta \gamma}\left(\underline{Y}_{\beta} \frac{\partial \omega}{\partial \underline{Y}_{\gamma}}+\underline{\omega}_{\beta k} \frac{\partial \omega}{\partial \underline{\omega}_{\gamma k}}\right)=0
$$

В соответствии с (46) термодинамический потенциал $\omega$ в обшем случае является функционалом следующих инвариантов: $Y_{0}, \underline{Y}^{2}, Y^{2}, Y_{4}, p^{2},(\underline{\omega} p)^{2},\left(\underline{\omega}_{k}\right)^{2}, Y_{k} p_{k}, Y_{k} \underline{\omega}_{\alpha k} \underline{Y}_{\alpha}$, $\left(\underline{Y}_{k}\right)^{2}, p_{k} \underline{\omega}_{\alpha k} \underline{Y}_{\alpha},\left(Y_{i} \underline{\omega}_{i}\right)^{2},\left(p_{i} \underline{\omega}_{\alpha i}\right)\left(Y_{k} \underline{\omega}_{\alpha k}\right)$.

\section{6. УРАВНЕНИЯ ГИДРОДИНАМИКИ}

Рассмотрим вопрос построения бездиссипативной гидродинамики В-фразы сверхтекучей ферми-жидкости. Такая жидкость на гидродинамическом этапе эволюции может быть описана сокрашенным набором параметров - плотностями аддитивных интегралов движения $\zeta_{a}(\mathbf{x})$, ортогональной матрицей поворота $a_{\alpha \beta}$ и сверхтекучей фазой $\theta(\mathbf{x})$ :

$$
\hat{f}(t) \underset{t \gg \tau_{0}}{\longrightarrow} \hat{f}(\zeta(t), a(t), \theta(t))
$$

где $\tau_{0}$ - время релаксации.

В обшем случае статистический оператор $\hat{f}=\hat{f}(\zeta, a, \theta)$ можно записать в виде $\hat{f}(\zeta, a, \theta)=\hat{f}_{0}(Y, a, \theta)+\hat{f}^{\prime}(\zeta, a, \theta)$, где $\hat{f}_{0}(Y, a, \theta) \equiv \hat{f}_{0}(\zeta(Y), a, \theta)$ - локально-равновесный статистический оператор, описываюший только обратимые процессы, a $\hat{f}^{\prime}$ - процессы релаксации.

Сформулируем уравнение для ортогональной матрицы поворота $a_{\alpha \beta} \cdot \mathrm{C}$ этой целью воспользуемся уравнением (6) и выражением (23):

$$
\dot{a}_{\alpha \beta}(\mathbf{x}, \hat{f})=i \operatorname{Sp} \hat{f}\left[\hat{\varepsilon}(\hat{f}), \hat{a}_{\alpha \beta}(\mathbf{x}, \hat{f})\right] .
$$

Пренебрегая релаксационными процессами в (53), имеем

$$
\dot{a}_{\alpha \beta}\left(\mathbf{x}, \hat{f}_{0}\right)=i \operatorname{Sp} \hat{f}_{0}\left[\hat{\varepsilon}\left(\hat{f}_{0}\right), \hat{a}_{\alpha \beta}\left(\mathbf{x}, \hat{f}_{0}\right)\right],
$$

откуда, используя соотношения (25), (40), получим уравнение движения для ортогональной матрицы поворота $a_{\alpha \beta}$ в главном приближении

$$
\dot{a}_{\alpha \beta}=a_{\alpha \lambda} \varepsilon_{\lambda \beta \gamma}\left(h_{\gamma}+\mathbf{q} \mathbf{v}_{n} n_{\gamma}\right), \quad h_{\gamma} \equiv-\frac{Y_{\gamma}}{Y_{0}}, \quad v_{n k} \equiv-\frac{Y_{k}}{Y_{0}} .
$$


Здесь $h_{\gamma}$ - внутреннее магнитное поле, $v_{n k}$ - нормальная скорость.

Аналогично выведем уравнение для сверхтекучей фазы. Согласно соотношению (31) уравнение движения для сверхтекучей фазы будет иметь вид

$$
\dot{\theta}(\mathbf{x}, \hat{f})=i \operatorname{Sp} \hat{f}[\hat{\varepsilon}(\hat{f}), \hat{\vartheta}(\mathbf{x}, \hat{f})] .
$$

Пренебрегая релаксационными процессами, имеем

$$
\dot{\theta}\left(\mathbf{x}, \hat{f}_{0}\right)=i \operatorname{Sp} \hat{f}_{0}\left[\hat{\varepsilon}\left(\hat{f}_{0}\right), \hat{\vartheta}\left(\mathbf{x}, \hat{f}_{0}\right)\right]
$$

откуда с учетом условия симметрии (40) и свойств (33) найдем уравнение движения для сверхтекучей фазы

$$
\dot{\theta}=\mu+\mathbf{p v}_{n}, \quad \mu \equiv-\frac{Y_{4}}{Y_{0}},
$$

где $\mu$ - химический потенциал. Уравнения движения для аддитивных интегралов движения согласно (6) имеют вид

$$
\dot{\zeta}_{a}(\mathbf{x}, \hat{f})=i \operatorname{Sp} \hat{f}\left[\hat{\varepsilon}(\hat{f}), \hat{\zeta}_{a}(\mathbf{x}, \hat{f})\right] .
$$

Пренебрегая релаксационными процессами, получим

$$
\dot{\zeta}_{a}\left(\mathbf{x}, \hat{f}_{0}\right)=i \operatorname{Sp} \hat{f}_{0}\left[\hat{\varepsilon}\left(\hat{f}_{0}\right), \hat{\zeta}_{a}\left(\mathbf{x}, \hat{f}_{0}\right)\right]=-\nabla_{i} \operatorname{Sp} \hat{f}_{0} \hat{\zeta}_{a i}\left(\mathbf{x}, \hat{f}_{0}\right),
$$

где вид плотностей потоков аддитивных интегралов движения $\zeta_{a k}$ в терминах локально-равновесного термодинамического потенциала определяется формулами $\left(47^{\prime}\right)$, (49), (51).

Таким образом, полная система уравнений гидродинамики сверхтекучей ферми-жидкости с триплетным спариванием в бездиссипативном приближении имеет вид

$$
\begin{aligned}
& \dot{\zeta}_{a}=-\nabla_{k} \underline{\zeta}_{a k}+\eta_{a}, \quad \dot{p}_{i}=\nabla_{i} p_{0}, \quad \underline{\dot{\omega}}_{\alpha k}=\nabla_{k} \underline{\omega}_{0 \alpha}+\eta_{\alpha k}, \\
& \eta_{a}=\delta_{a \alpha} \varepsilon_{\alpha \beta \gamma}\left(\underline{\zeta}_{\gamma k} \underline{\omega}_{\beta k}+\underline{\zeta}_{\gamma} \underline{\omega}_{\beta 0}\right),
\end{aligned}
$$

Следствием уравнений (59) является адиабатичность течения рассматриваемой сверхтекучей жидкости: $\dot{\sigma}=-\nabla_{i}\left(\sigma v_{n k}\right)$, где $\sigma=-\omega+Y_{a} \zeta_{a}$ - плотность энтропии.

Если термодинамический потенциал $\omega$ в (59) является функцией только термодинамических параметров $p_{i}, Y_{i}, Y_{4}$ (т.е. из набора переменных исключены термодинамические параметры, описываюшие магнитные свойства системы - $\left.\underline{\omega}_{\alpha i}, Y_{\alpha}\right)$, то уравнения примут вид уравнений гидродинамики сверхтекучей ферми-жидкости с синглетным спариванием $[27,28,49]$. Если же $\omega$ является функцией только термодинамических параметров $\underline{\omega}_{\alpha i}, Y_{\alpha}$ (из набора термодинамических параметров исключены переменные $\left.p_{i}, Y_{i}, Y_{4}\right)$, то полученные уравнения перейдут в уравнения для неупорядоченных или многоподрешеточных магнетиков $[30,31]$.

При конкретном модельном виде плотности энергии, выбираемом как предложено в работах [6-8], получаются результаты, согласуюшиеся с этими работами. 


\section{7. ГАЛИЛЕЕВО- И РЕЛЯТИВИСТСКИ-ИНВАРИАНТНЫЕ УРАВНЕНИЯ ГИДРОДИНАМИКИ}

При преобразованиях Галилея, определяемых унитарным преобразованием $U_{\mathbf{v}_{0}}=$ $\exp \left(-i \mathbf{v}_{0} \int d^{3} x \mathbf{x} \hat{n}(\mathbf{x})\right)$, операторы плотностей адлитивных интегралов движения обладают согласно (5) следуюшими трансформационными свойствами:

$$
\begin{gathered}
U_{\mathbf{v}_{0}}^{+} \hat{n}(\mathbf{x}) U_{\mathbf{v}_{0}}=\hat{n}(\mathbf{x}), \quad U_{\mathbf{v}_{0}}^{+} \hat{s}_{\alpha}(\mathbf{x}) U_{\mathbf{v}_{0}}=\hat{s}_{\alpha}(\mathbf{x}), \quad U_{\mathbf{v}_{0}}^{+} \hat{\pi}_{k}(\mathbf{x}) U_{\mathbf{v}_{0}}=\hat{\pi}_{k}(\mathbf{x})+m v_{0 k} \hat{n}(\mathbf{x}), \\
U_{\mathbf{v}_{0}}^{+} \hat{\varepsilon}\left(\mathbf{x}, U_{\mathbf{v}_{0}} \hat{f} U_{\mathbf{v}_{0}}^{+}\right) U_{\mathbf{v}_{0}}=\hat{\varepsilon}(\mathbf{x}, \hat{f})+v_{0 k} \hat{\pi}_{k}(\mathbf{x})+\left(m v_{0}^{2} / 2\right) \hat{n}(\mathbf{x}),
\end{gathered}
$$

где $\mathbf{v}_{0}$ - параметр преобразования, $m$ - масса ферми-частицы. Последнее из этих соотношений имеет место только для галилеево-инвариантного функционала энергии. При этом термодинамический потенциал $\omega$ будет зависеть от меньшего числа инвариантов: $Y_{0}^{\prime}, \underline{Y}^{2}, Y^{\prime 2}, Y_{4}^{\prime}, \underline{\omega}_{k}^{2}, Y_{k}^{\prime} \underline{\omega}_{\alpha k} \underline{Y}_{\alpha},\left(\underline{Y} \underline{\omega}_{k}\right)^{2},\left(Y_{i}^{\prime} \underline{\omega}_{i}\right)^{2}$, здесь $Y_{0}^{\prime}=Y_{0}, \quad Y_{i}^{\prime}=Y_{i}+Y_{0} v_{0 i}$, $Y_{4}^{\prime}=Y_{4}+Y_{k} m v_{0 k}+Y_{0}\left(m v_{0}^{2} / 2\right), v_{0 k}=p_{k} / m$.

Рассмотрим теперь уравнения (59) в случае, когда система является релятивистски-инвариантной. Это удобно сделать, переходя к релятивистским тензорным величинам. Введем плотности аддитивных интегралов движения и соответствуюшие им плотности потоков в состоянии локального термодинамического равновесия в терминах потенциала Гиббса $\omega^{\prime} \equiv \omega / Y_{0}$, представляющего собой релятивистский скаляр:

$$
t^{\mu \nu}=-\frac{\partial\left(Y^{\nu} \omega^{\prime}\right)}{\partial Y_{\mu}}+p^{\mu} j^{\nu}+\underline{\omega}_{\alpha}^{\mu} \underline{j}_{\alpha}^{\nu}, \quad j^{\nu}=\frac{\partial \omega^{\prime}}{\partial p_{\nu}}, \quad \underline{j}_{\alpha}^{\nu}=\frac{\partial \omega^{\prime}}{\partial \underline{\omega}_{\alpha \nu}},
$$

здесь $t^{\mu \nu} \equiv\left(t^{00}=\varepsilon, t^{0 k}=w_{k}, t^{k 0}=\pi_{k}, t^{k l}=t_{k l}\right)$ - 4-тензор энергии-импульса, $j^{\nu} \equiv\left(n, j_{k}\right)$ - 4-ток заряда, $\underline{j}_{\alpha}^{\nu} \equiv\left(\underline{s}_{\alpha}, \underline{j}_{\alpha k}\right)-4$-ток изоспина, $p^{\nu} \equiv\left(p_{0}, p_{k}\right)$ - сверхтекучий 4-импульс, $\underline{\omega}_{\alpha}^{\nu} \equiv\left(\underline{\omega}_{0 k}, \underline{\omega}_{\alpha k}\right)-4$-форма Картана, $Y_{\mu}=\left(Y_{0}, Y_{i}\right)$. Гиббсовский потенциал $\omega^{\prime}$ является функционалом семи инвариантов: $Y^{2}, p^{2}, \underline{\omega}^{2}, Y p,(Y \underline{\omega})^{2},(p \underline{\omega})^{2}$, $(Y \underline{\omega})(p \underline{\omega})$.

Уравнения идеальной гидродинамики сверхтекучей ферми-жидкости с триплетным спариванием в релятивистски-инвариантной форме можно записать в виде

$$
\begin{gathered}
\nabla_{\nu} t^{\mu \nu}=0, \quad \nabla_{\nu} j^{\nu}=0, \quad \nabla_{\nu} \underline{j}_{\alpha}^{\nu}=\varepsilon_{\alpha \beta \gamma} \underline{\omega}_{\beta \mu} \underline{j}_{\gamma}^{\mu}, \\
\nabla^{\mu} p^{\nu}-\nabla^{\nu} p^{\mu}=0, \quad \nabla^{\mu} \underline{\omega}_{\alpha}^{\nu}-\nabla^{\nu} \underline{\omega}_{\alpha}^{\mu}=-\varepsilon_{\alpha \beta \gamma} \underline{\omega}_{\beta}^{\nu} \underline{\omega}_{\gamma}^{\mu} .
\end{gathered}
$$

Первые три уравнения включают в себя уравнения движения для плотностей аддитивных интегралов движения, два последних - уравнения движения для сверхтекучего импульса $p_{k}$ и формы Картана $\underline{\omega}_{\alpha k}$, а также условие потенциальности и тождество Маурера-Картана.

Введя 4-ток энтропии $\sigma^{\mu} \equiv\left(\sigma, \sigma_{k} \equiv \sigma v_{n k}\right)$, можно записать второй закон термодинамики в релятивистской форме

$$
d \sigma^{\mu}=Y_{\nu} d t^{\nu \mu}+Y_{4} d j^{\mu}+\underline{Y}_{\alpha} d \underline{j}_{\alpha}^{\mu}-\left(Y^{\mu} j^{\nu}-Y^{\nu} j^{\mu}\right) d p_{\nu}-\left(Y^{\mu} \underline{j}_{\alpha}^{\nu}-Y^{\nu} \underline{j}_{\alpha}^{\mu}\right) d \underline{\omega}_{\alpha \nu},
$$

где 4-ток энтропии $\sigma^{\mu}$ удовлетворяет условию адиабатичности

$$
\nabla_{\mu} \sigma^{\mu}=0, \quad \sigma^{\mu}=-Y^{\mu} Y_{\nu} \frac{\partial \omega^{\prime}}{\partial Y_{\nu}} .
$$


Уравнения этого раздела могут быть применимы в астрофизике и других разделах физики, рассматривающих сверхтекучую релятивистскую жидкость с триплетным спариванием.

\section{8. СПЕКТРЫ СОБСТВЕННЫХ КОЛЕБАНИЙ СВЕРХТЕКУЧЕЙ ФЕРМИ-ЖИДКОСТИ С ТРИПЛЕТНЫМ СПАРИВАНИЕМ}

Рассмотрим спектры собственных колебаний в такой жидкости. Линеаризуя систему уравнений (59) вблизи состояния равновесия и переходя в них к фурье-компонентам, получим дисперсионное уравнение

$$
\operatorname{det}\left(\hat{A}+\widehat{B} \underline{\widehat{B}}^{-1} \underline{\hat{A}}\right)=0
$$

где входящие в уравнение (64) матрицы $\hat{A}, \underline{\hat{A}}, \widehat{B}, \underline{\widehat{B}}$ имеют вид

$$
\begin{gathered}
A_{a^{\prime} b}=i\left(\omega \delta_{a^{\prime} b}-k_{i} \underline{\zeta}_{a^{\prime} i ; b}\right), \quad B_{a^{\prime} 4}=k_{i} k_{j} \underline{\zeta}_{a^{\prime} i ; j}, \quad B_{a^{\prime} \beta}=k_{i}\left(k_{j} \delta_{\alpha \beta}-i q_{j} \xi_{\alpha \beta}\right) \underline{\zeta}_{a^{\prime} i ; \alpha j} \\
A_{\alpha b}=i\left(\omega \delta_{\alpha b}-k_{i} \underline{\zeta}_{\alpha i ; b}\right)-\eta_{\alpha ; b}, \quad B_{\alpha 4}=k_{i} k_{j} \underline{\zeta}_{\alpha i ; j}+i \eta_{\alpha ; j} k_{j}, \\
B_{\alpha \beta}=k_{i}\left(k_{j} \delta_{\lambda \beta}-i q_{j} \xi_{\lambda \beta}\right) \underline{\zeta}_{\alpha i ; \lambda j}+\eta_{\alpha ; \lambda j}\left(k_{j} \delta_{\lambda \beta}-i q_{j} \xi_{\lambda \beta}\right), \\
A_{4 b}=i k^{2} p_{0 ; b}, \quad B_{44}=k^{2}\left(\omega+k_{j} p_{0 ; j}\right), \quad B_{4 \beta}=k^{2}\left(k_{j} \delta_{\alpha \beta}-i q_{j} \xi_{\alpha \beta}\right) p_{0 ; \alpha j} \\
\underline{A}_{\alpha b}=i k^{2} \underline{\omega}_{\alpha 0 ; b}-k_{i} \eta_{\alpha i ; b}, \quad \underline{B}_{\alpha 4}=k^{2} k_{j} \underline{\omega}_{\alpha 0 ; j}+i k_{i} k_{j} \eta_{\alpha i ; j} \\
\underline{B}_{\alpha \beta}=\omega\left(k^{2} \delta_{\alpha \beta}-i k_{j} q_{j} \xi_{\alpha \beta}\right)+\left(k^{2} \underline{\omega}_{\alpha 0 ; \lambda j}+i k_{i} \eta_{\alpha i ; \lambda j}\right)\left(k_{j} \delta_{\lambda \beta}-i q_{j} \xi_{\lambda \beta}\right) .
\end{gathered}
$$

Здесь $c_{m ; a}=\partial c_{m} / \partial \underline{\zeta}_{a}, c_{m ; i}=\partial c_{m} / \partial p_{i}, c_{m ; \alpha i}=\partial c_{m} / \partial \underline{\omega}_{\alpha i}, c_{m} \equiv\left(\underline{\zeta}_{a k}, p_{0}, \underline{\omega}_{\alpha 0}\right.$, $\left.\eta_{\alpha}, \eta_{\alpha k}\right)$. Потоки, входящие в матрищы $A, B, \underline{A}, \underline{B}$, выражаются через плотность термодинамического потенциала.

Дисперсионное уравнение (64) в обшем случае представляет собой уравнение двенадцатой степени по частоте, которое сложно решить аналитически. Поэтому мы рассмотрим несколько частных случаев. Для дальнейшего рассмотрения удобно представить плотности потоков аддитивных интегралов движения, входящих в матричные элементы $A, B, \underline{A}, \underline{B}$, в терминах плотности функционала энергии, а также заменить уравнение для плотности энергии уравнением для энтропии. Если функционал энергии зависит только от $\zeta_{4}, \sigma, \zeta_{\alpha}^{2}, \zeta_{i}^{2}, p^{2}, \omega_{k}^{2}, p_{i} \zeta_{i},(\omega p)^{2}$ и все векторные величины в равновесии обрашаются в нуль, то решениями дисперсионного уравнения будут выражения

$$
\begin{aligned}
\omega^{2} & =c_{ \pm}^{2} k^{2}, \quad \omega_{n}^{2}=c_{s}^{2} k^{2}, \quad n=1,2,3, \\
c_{ \pm}^{2} & =\frac{1}{2}\left(Q \pm \sqrt{Q^{2}+4 Q^{\prime}}\right), \quad c_{s}=R,
\end{aligned}
$$

где

$$
\begin{gathered}
Q=\sigma^{0}\left(a_{2} b_{1}+c_{1} a_{3}\right)+a_{1}\left(a_{2}+\zeta_{4}^{0} a_{3}\right)+c_{2}\left(a_{3}+\zeta_{4}^{0} a_{4}\right), \\
Q^{\prime}=\sigma^{0}\left(c_{2} b_{1}-c_{1} a_{1}\right)\left(a_{4} a_{2}-a_{3}^{2}\right), \quad R=c_{3} b_{2}, \quad a_{1}=\partial^{2} \varepsilon / \partial \zeta_{4}^{2}, \\
a_{2}=2 \partial \varepsilon / \partial p^{2}, \quad a_{3}=\partial \varepsilon / \partial\left(p_{i} \zeta_{i}\right), \quad a_{4}=2 \partial \varepsilon / \partial \pi^{2}, \quad b_{1}=\partial^{2} \varepsilon / \partial \zeta_{4} \partial \sigma \\
c_{1}=\partial p / \partial \sigma, \quad c_{3}=2 \partial \varepsilon / \partial \zeta_{\alpha}{ }^{2}, \quad b_{2}=2 \partial \varepsilon / \partial \underline{\omega}^{2}, \quad c_{2}=\partial p / \zeta_{4}, \quad p=-\omega / Y_{0} .
\end{gathered}
$$


Выражения (66) соответствуют по форме первому, второму звукам и спиновым волнам $[5,9,10]$. Если же отличен от нуля только сверхтекучий импульс, то решения в случае $\mathbf{p} \perp \mathbf{k}$ имеют вид

$$
\begin{gathered}
\omega^{2}=c_{1 \pm}^{2} k^{2}, \quad \omega_{n}^{2}=c_{s}^{2} k^{2}, \quad n=1,2,3, \\
c_{1 \pm}^{2}=\frac{1}{2}\left(Q_{1} \pm \sqrt{Q_{1}^{2}+4 Q_{1}^{\prime}}\right) .
\end{gathered}
$$

Здесь $\left.Q_{1}=-Q-a_{4} a_{2} p^{2}, \quad Q_{1}^{\prime}=-Q^{\prime}+a_{4} c_{2}\left[a_{2}\left(a_{3}+\zeta_{4}^{0} a_{4}\right)+a_{3} a_{2}+\zeta_{4}^{0} a_{3}\right)\right]+$ $\sigma^{0} a_{4} c_{1}\left(a_{3}^{2}+a_{4} a_{2}\right) p^{2}$.

Учтем взаимодействие орбитальной и спиновой подсистем. С этой целью в функционал энергии, приведенный выше, добавим слагаемое вида $f \underline{s}_{\alpha} \underline{\omega}_{\alpha k} \pi_{k}$ и будем считать, что только вектор магнитной спирали отличен от нуля. В случае, если $\mathbf{q} \perp \mathbf{k}$, решения примут следующий вид:

$$
\begin{gathered}
\omega^{2}=c_{ \pm}^{2} k^{2}, \quad \omega_{1}^{2}=\left(c_{s}^{2}+c_{q}^{2} q^{2}\right) k^{2}, \quad \omega_{2,3}^{2}=c_{s}^{2}\left(k^{2} \pm \mathbf{q k}\right) \\
c_{q}^{2}=4 b_{2}\left(a_{4}+f\right) .
\end{gathered}
$$

При рассмотрении спиновой динамики, считая влияние орбитальной подсистемы слабым, мы получим спектры, качественно сходные с результатами работы [30]. Приведем только некоторые из них:

$$
\begin{array}{cc}
s^{0} \neq 0, \quad h^{0} \neq 0, \quad q^{0}=0, \quad \omega_{1}^{2}=\left(v^{\prime}+v^{\prime \prime}\right)^{2} k^{2}, \quad \omega_{2}^{2}=\left(v^{\prime}-v^{\prime \prime}\right)^{2} k^{2}, \quad \omega_{3}^{2}=\left(\omega_{0}+r k^{2}\right)^{2} ; \\
s^{0} \neq 0, \quad h^{0} \neq 0, \quad q^{0} \neq 0, \quad \omega_{1,2}=\left(v^{\prime}+v^{\prime \prime}\right) k, \quad \omega_{3,4}=\left(u^{\prime}+u^{\prime \prime}\right) k, \\
\omega_{5,6}=\omega_{0} \pm r^{\prime} k+r^{\prime \prime} k^{2} .
\end{array}
$$

Коэффициенты при волновом векторе и $\omega_{0}$ выражаются в терминах функционала энергии. Как видно из вышесказанного, формулы (66)-(68) отражают безактивационные спектры, в то время как (69) - спектры с активацией. Спектры (67)-(69) отличаются от получавшихся ранее $[5,16,50]$ наличием ненулевых значений вектора спирали, сверхтекучего импульса и спина в состоянии равновесии.

\section{Список литературы}

[1] Л. Д. Ландау. Сб. трудов. Т. 1. М.: Наука, 1969. С. 128-143, 352-385.

[2] Н. Н. Боголюбов. Избр. труды. Т. 3. Киев: Наукова думка, 1971. С. 11-17, 244-281.

[3] И. М. Халатников. Теория сверхтекучести. М.: Наука, 1971.

[4] С. Паттерман. Гидродинамика сверхтекучей жидкости. М.: Мир, 1978.

[5] A. J. Leggett. Rev. Mod. Phys. 1975. V. 47. P. 331.

[6] R. Combescot. J. Phys. C. 1981. V. 14. P. 1619.

[7] P. Wölfle. Phys. Rev. B. 1976. V. 14. P. 89.

[8] K. Maki, P. Kumar. Phys. Rev. B. 1977. V. 16. P. 182.

[9] G. E. Volovik. Exotic properties of superfluid ${ }^{3}$ He. Singapore: World Sci. Pub., 1992.

[10] D. Vollhardt, P. Wölfle. The Superfluid Phases of Helium 3. Ed. Taylor and Francis. London, 1990.

[11] В. П. Минеев. УФН. 1983. Т. 139. С. 303.

[12] В. П. Минеев, Г. Е. Воловик. ЖЭТФ. 1977. Т. 72. С. 2256.

[13] Г. Е. Воловик. Письма в ЖЭТФ. 1984. Т. 39. С. 304.

[14] H. Kleinert. Phys. Lett. A. 1979. V. 71. P. 66.

[15] M. Liu. Phys. Rev. B. 1976. V. 13. P. 4174. 
[16] R. Graham, H. Pleiner. J. Phys. C. 1976. V. 9. P. 279.

[17] W. F. Brinkman, M. C. Cross. In: Progr. in Low Temp. Phys. V. 7, 1978. P. 105-170.

[18] M. Liu, M. C. Cross. Phys. Rev. Lett. 1978. V. 41. P. 250.

[19] R. Combescot, T. Dombre. Phys. Lett. A. 1980. V. 76. P. 293.

[20] Д. Н. Зубарев. Неравновесная статистическая термодинамика. М.: Наука, 1971.

[21] А.И. Ахиезер, С. В. Пелетминский. Методы статистической физики. М.: Наука, 1977.

[22] В. Г. Морозов. ТМФ. 1976. Т. 28. С. 267.

[23] М. Ю. Ковалевский, Н. М. Лавриненко, А.И. Соколовский, С.В. Пелетминский. TMФ. 1982. Т. 50. C. 450.

[24] Ю. А. Церковников. ТМФ. 1992. Т. 93. С. 412.

[25] Н.Н. Боголюбов(мл.), М.Ю. Ковалевский, А.М. Курбатов, С. В. Пелетминский, A. H. Тарасов. УФН. 1989. Т. 159. С. 585.

[26] Н. Н. Боголюбов. Квазисредние в задачах статистической механики. Препринт ОИЯИ Д-781. Дубна: ОИЯИ, 1961.

[27] В.В. Красильников, С.В. Пелетминский, А.А. Рожков, А.А. Яченко. ЭЧАЯ. 1988. T. 19. C. 1440.

[28] В. В. Красильников, А. А. Рожков, А. А. Яченко. ФНТ. 1990. Т. 16. С. 1368.

[29] А. П. Ивашин, В. В. Красильников, А. А. Рожков. ФНТ. 1995. Т. 21. С. 58.

[30] M. Yu. Kovalevskii, A. A. Rozhkov. Physica. 1995. V. 216. P. 169.

[31] М. Ю. Ковалевский, С. В. Пелетминский. ТМФ. 1994. Т. 100. С. 59.

[32] Н. Н. Боголюбов. ДАН СССР. 1958. Т. 119. С. 52.

[33] А. Б. Мигдал. ЖЭТФ. 1959. Т. 37. С. 249.

[34] W. Israel. Phys. Lett. A. 1981. V. 86. P. 79.

[35] В. В. Лебедев, И. М. Халатников. ЖЭТФ. 1982. Т. 83. С. 1601.

[36] П. И. Фомин, В. И. Шадура. Препринт ИТФ-83-150Р. Киев: ИТФ, 1983.

[37] W. G. Dixon. Arch. Rat. Mech. Anal. 1982. V. 80. P. 159.

[38] W. Israel. Phys. Lett. A. 1982. V. 92. P. 71.

[39] А. В. Прозоркевич, В. Л. Самородов, С. А. Смолянский. ТМФ. 1982. Т. 52. С. 463.

[40] С. А. Смолянский. ТМФ. 1982. Т. 52. С. 292.

[41] М.Ю. Ковалевский, С.В. Пелетминский, А.Н. Тарасов, П.И. Фомин. Препринт ИТФ-83-151Р. Киев: ИТФ, 1983.

[42] Дж. С. Цакадзе, С. Дж. Цакадзе. УФН. 1975. Т. 115. С. 503.

[43] Я. Б. Зельдович, И. Д. Новиков. Строение и эволюция Вселенной. М.: Наука, 1975.

[44] A. D. Linde. Rep. Progr. Phys. 1979. V. 42. P. 389.

[45] Л. Иииксон, Ж.-Б. Зюбер. Квантовая теория поля. М.: Мир, 1984.

[46] A. Картан. Геометрия групп Ли и симметрические пространства. М.: ИИЛ, 1949.

[47] N. D. Mermin, T. L. Ho. Phys. Rev. Lett. 1975. V. 36. P. 594.

[48] Г. Е. Воловик. УФН. 1984. Т. 143. С. 73.

[49] М.Ю. Ковалевский, В.А. Красников, С.В. Пелетминский. Докл. АН СССР. 1988. T. 303. C. 337.

[50] В. В. Лебедев. ЖЭТФ. 1979. Т. 76. С. 257.

Поступила в редакцию 5.XII. 1995 г., после доработки 4.VII.1997 г.

\section{Yu. Kovalevskii, A. A. Rozhkov \\ ON THE THEORY OF B-PHASE IN THE SUPERFLUID FERMI-LIQUID WITH TRIPLET PAIRING}

Thermodynamics of superfluid Fermi-liquid with triplet pairing is constructed and hydrodynamic equations are derived. Parameters of abridged description such as superfluid phase and orthogonal rotation matrix are represented in the terms of the order parameter. Theorem of additive motion integral connection is generalized for the case of local equilibrium. Galilean and Lorentz invariant physical systems are considered in detail. Spectra of collective excitations of considered Fermi-liquid are obtained. 